\title{
Gas Outburst Prediction Model Using Improved Entropy Weight Grey Correlation Analysis and IPSO-LSSVM
}

\author{
Haibo Liu $\left(\mathbb{D},{ }^{1}\right.$ Yujie Dong $(\mathbb{D})^{2}$ and Fuzhong Wang ${ }^{1}{ }^{1}$ \\ ${ }^{1}$ School of Electric Engineering and Automation, Henan Polytechnic University, Jiaozuo 454000, China \\ ${ }^{2}$ College of Computer Science and Technology, Henan Polytechnic University, Jiaozuo 454000, China
}

Correspondence should be addressed to Haibo Liu; liuhaibo09@hpu.edu.cn

Received 26 August 2020; Revised 10 October 2020; Accepted 30 October 2020; Published 16 November 2020

Academic Editor: M Syed Ali

Copyright (c) 2020 Haibo Liu et al. This is an open access article distributed under the Creative Commons Attribution License, which permits unrestricted use, distribution, and reproduction in any medium, provided the original work is properly cited.

\begin{abstract}
This paper investigates the problem of gas outburst prediction in the working face of coal mine. Firstly, based on a comprehensive analysis of influence factors of gas outburst, an improved entropy weight algorithm is introduced into a grey correlation analysis algorithm; thus, the reasonable weights and correlation order of the influencing factors are obtained to improve the objectivity of the evaluation. The main controlling factors obtained are used as the input of the prediction model. Secondly, by utilizing the improved particle swarm optimization (IPSO), the penalty factor and kernel parameter of least square support vector machine (LSSVM) are optimized to enhance the global search ability and avoid the occurrence of the local optimal solutions, and a new prediction model of gas outburst based on IPSO-LSSVM is established. At last, the prediction model is applied in the tunneling heading face 14141 of Jiuli Hill mine in Jiaozuo City, China. The case study demonstrates that the prediction accuracy of the proposed model is $92 \%$, which is improved compared with that of the SVM model and GA-LSSVM model.
\end{abstract}

\section{Introduction}

Gas outburst is a kind of complicated dynamic phenomenon in the process of coal mining [1]. A large amount of gas is ejected from gas-bearing coal rock to a mining face with rapid speed in a very short time. China is one of the most seriously stricken by gas outburst and gas explosion countries in the world. As the mining depth continues to increase, the gas outburst is becoming increasingly serious, especially in the case of high geostress and gas pressure conditions [2]. It gravely threatens the health and safety of miners, damages facilities, and decreases coal production. Therefore, the scientific and accurate prediction of gas outburst is very important for the safety of coal mine production.

Over the past years, gas outburst prediction research has achieved fruitful results [3-20]. Numerous approaches are currently used to predict the gas outburst. Roughly speaking, these methods can be classified into two types: classical prediction method and artificial intelligence prediction method. Classical prediction methods use single-factor or multifactor comprehensive effects to predict from the outburst mechanism, mainly including $\mathrm{D}-\mathrm{K}$ comprehensive factor prediction, geodynamic division, and other methods $[3,4]$. Since these methods are relatively simple in theory, a large amount of experimental data are required. However, the data acquisition process is complicated and requires high time and economic costs. With the rapid development of data mining technology and machine learning, some methods such as data mining algorithms $[5,6]$, neural networks [7], D-S evidence theory [8], rough set theory [9], machine learning algorithms [10-12], support vector machines (SVM) [13-15], and wavelet transform [16, 17] have been gradually adopted in the field of gas outburst prediction. In [6], BP neural network and D-S theory were used to establish the gas outburst prediction model: the BP neural network was optimized by PSO in the feature layer and D-S theory was used to make the fusion decision of time domain and space domain. A PCA-BP neural network prediction model was established; three principal components were extracted through the PCA method [7]. In [8], a gas outburst evaluation strategy was established by using the combination 
of fuzzy neural network and D-S theory, and the outburst level was accurately predicted. In [9], by combining artificial neural network (ANN) and coupled fault tree analysis (FTA), the prediction model was proposed to improve the prediction of the potential risk of gas outburst events. The eight main model parameters identified by the FTA method were used as input variables to an ANN model. In order to overcome traditional GABP mode problems, an effective genetic algorithm was proposed to reduce its iterative calculation time, and the parameters of BPNN could be determined automatically, so as to enhance the training ability of BPNN [12]. In [14], a decision table of gas outburst intensity was established by employing the RS theory and SVM, using the attribute reduction algorithm in rough set theory and PSO algorithm to optimize parameters of SVM, which improved the prediction accuracy. All of the above studies have proposed their own methods in the prediction of gas outbursts and have achieved good results but also have certain limitations. For example, neural networks are prone to local minima and overfitting for small sample problems. Moreover, there are problems such as dimensional disaster and network structure. In addition, the selection of kernel function and penalty parameters of the traditional SVM model is difficult. Therefore, it is necessary to introduce a new effective method to improve gas outburst prediction accuracy.

Motivated by the above discussion, in this paper, we will study the prediction factors analysis through the improved entropy weight grey correlation and IPSO-LSSVM prediction model. The main contributions can be summarized as follows:

(1) Based on a comprehensive analysis of the influence factors of gas outburst, an improved entropy weight algorithm is introduced into a grey relational analysis algorithm; thus the reasonable weights and correlation order of influencing factors are obtained to improve the objectivity of the evaluation.

(2) By using IPSO to optimize the punishment factor and nuclear radial range of LSSVM, the gas outburst prediction model based on IPSO-LSSVM is established, which can better consider that the influencing factors have a comprehensive effect on outburst risk and improve the accuracy of gas outburst prediction.

The rest of this paper is organized as follows: In Section 2 , the main controlling factors influencing gas outburst analysis are presented. In Section 3, the LSSVM method and the IPSO method are adopted to establish the gas outburst prediction model. Application of the IPSO-LSSVM model in gas outburst prediction is given in Section 4, and conclusions follow in Section 5.

\section{Materials and Methods}

2.1. Identify the Main Controlling Factors Influencing Gas Outburst. Grey system is generally used to study the uncertainty problem of the small sample, poor information, and multiple factors. According to the entropy weight method, the smaller the information entropy of factor, the greater the variation degree of factor value, the greater the role it plays in the analysis, and the greater its weight [18]. The entropy weighted grey correlation method is concise and clear, with low data requirements and small calculation amount, which is suitable for analyzing the main controlling factors.

2.1.1. Improved Entropy Weight Method. The entropy weight method uses the information entropy to measure the information utility value of each evaluation factor and determine the entropy weight, which is an objective weighting method. The steps of the improved entropy weight method are as follows:

(1) Establishing the original evaluation matrix $R=\left(r_{i j}\right)_{m \times n}$.

Suppose there are $n$ evaluation factors and $m$ items to be evaluated, where $r_{i j}$ is the evaluation value of the $i$-th item under the $j$-th factor:

$$
R_{i j}=\left[\begin{array}{cccc}
r_{11} & r_{12} & \cdots & r_{1 n} \\
r_{21} & r_{22} & \cdots & r_{2 n} \\
\vdots & \vdots & & \vdots \\
r_{m 1} & r_{m 2} & \cdots & r_{m n}
\end{array}\right] .
$$

(2) Normalize the original evaluation matrix and get the normalized matrix $R^{\prime}=\left(r_{i j}^{\prime}\right)_{m \times n}$.

Since the dimensions of each evaluation factor are different and cannot be directly analyzed and compared, the per-unit value is used to perform dimensionless processing on each factor. For the positive factor,

$$
r_{i j}^{\prime}=\frac{r_{i j}-r_{\min }}{r_{\max }-r_{\min }} .
$$

For the negative factor,

$$
r_{i j}^{\prime}=1-\frac{r_{i j}-r_{\min }}{r_{\max }-r_{\min }},
$$

where $r_{\min }$ and $r_{\max }$ are the minimum and maximum values of the factor, respectively.

(3) Calculating entropy value and entropy weight of evaluation factor,

$$
H_{j}=\left\{\begin{array}{l}
-\frac{1}{\ln n} \sum_{i=1}^{m} P_{i j} \operatorname{In} P_{i j} \quad P_{i j} \neq 0, \\
0 \quad P_{i j}=0,
\end{array}\right.
$$

where

$$
P_{i j}=r_{i j}^{\prime} / \sum_{i=1}^{m} r_{i j}^{\prime} \quad(i=1,2, \cdots, m ; j=1,2, \cdots, n) .
$$

The traditional entropy weight method uses a standardized processing method when calculating the entropy weight $\omega_{j}$, as shown in the following equation: 


$$
\omega_{j}=\frac{1-H_{j}}{\sum_{j=1}^{n}\left(1-H_{j}\right)}=\frac{1-H_{j}}{n-\sum_{j=1}^{n} H_{j}} .
$$

It can be seen from the above equation that when $H_{j} \longrightarrow 1(j=1,2, \ldots, n)$, the difference in the entropy value of different evaluation indexes is small, but the difference in the entropy weight is large. Therefore, this paper uses the following equation to calculate entropy weight instead of equation (5):

$$
\omega_{j}^{\prime}=\frac{\sum_{k=1}^{n} H_{k}+1-H_{j}}{\sum_{l=1}^{n}\left(\sum_{k=1}^{n} H_{k}+1-2 H_{l}\right)} .
$$

In the above method, the different entropy weights for different factors (e.g., $p$ and $q$ ) become

$$
\Delta \omega^{\prime}=2 \Delta H / C \text {, }
$$

where $\Delta H=H_{q}-H_{p}, C=\sum_{l=1}^{n}\left(\sum_{k=1}^{n} H_{k}+1-2 H_{l}\right)$. When $|\Delta H|<\varepsilon,\left|\Delta \omega^{\prime}\right|<2 \cdot \varepsilon / C$, it indicates that the entropy value changes slightly, and its corresponding entropy weight also changes slightly. For the factor with equivalent useful information, the weight information is consistent with the corresponding entropy level.

\subsubsection{Improved Entropy Weight Grey Correlation Analysis} Algorithm. Grey relational analysis (GRA) is a multifactor statistical analysis method. Compared with other theories, it has an incomparable role in the research of the small sample and poor information uncertainty. Its principle is to use the similarity between the change curve of the object to be analyzed and the ideal data curve to determine the level of correlation between the two, and it is easy to handle the correlation between elements with uncertain and incomplete information [16]. The grey correlation degree is calculated as follows:

(1) Determine the reference and comparison columns, let $X_{i}=\left\{x_{i}(k) \mid k=1,2, \cdots, n\right\}$ be the comparison column and $X_{0}=\left\{x_{0}(k) \mid k=1,2, \cdots, n\right\}$ be the reference column.

(2) Dimensionless processing of data.

(3) Calculate the absolute difference: select a reference column, assuming that the absolute difference between $x_{i}$ and $x_{0}$ at each time is $\delta$, $\delta=\left|x_{0}(k)-x_{i}(k)\right|, k=1,2, \cdots, n$.

(4) Calculation of grey correlation coefficient: the correlation coefficient between the $k$-th index and $k$-th optimal index value of the reference column is derived as follows:

$$
\varphi_{i}(k)=\frac{\min _{i} \min _{k}\left|x_{0}(k)-x_{i}(k)\right|+\lambda \max _{i} \max _{k}\left|x_{0}(k)-x_{i}(k)\right|}{\left|x_{0}(k)-x_{i}(k)\right|+\lambda \max _{i} \max _{k}\left|x_{0}(k)-x_{i}(k)\right|},
$$

where $\lambda$ is the resolution coefficient, $\lambda \in(0,1)$. This coefficient is introduced to improve the significance of the difference between the correlation coefficients.
(5) Calculation of grey correlation degree:

$$
\mu_{i}=\frac{1}{n} \sum_{k=1}^{n} \varphi_{j}(k)
$$

In order to reduce the impact of correlation coefficient and human subjectivity, the entropy weight method is introduced to objectively weigh each influencing factor, and the improved grey relational entropy (GRE) method is proposed to remove the subjective impact while improving the calculation accuracy of the grey relational analysis. The grey correlation entropy is calculated as follows:

$$
\mu_{i}=\frac{1}{n} \sum_{k=1}^{n} \omega_{j}^{\prime} \varphi_{j}(k)
$$

The influencing factors are sorted according to the correlation degree $\mu_{i}$, and the influencing factors with the large correlation degree have a greater impact on the reliability factors.

\section{Establishment of the IPSO-LSSVM Prediction Model}

3.1. Least Square Support Vector Machine (LSSVM). SVM has great advantages compared with other methods in the gas outburst prediction. Its characteristic is to construct the algorithm based on the principle of structural risk minimization, which has good generalization ability and can obtain the global optimal solution [15]. However, the standard SVM algorithm needs to solve the quadratic programming problem, which requires a large amount of calculation. LSSVM uses the least squares algorithm instead of the quadratic optimization algorithm in SVM and transforms the convex quadratic programming problem into a solution of linear equations, which simplifies the calculation process and improves the learning speed [13, 21, 22].

The principle of LSSVM is to use a nonlinear function to transform the input vector to a high-dimensional feature space and construct a linear function in the high-dimensional feature space to describe the nonlinear relationship between the input vector and the output variable to minimize structural risk. Set the training sample set $L=\left\{\left(x_{i}, y_{i}\right) \mid i=1,2, \cdots, n\right\}, x_{i} \in R^{d}$ is the d-dimension input vector, and $y_{i} \in R$ is the corresponding output vector. $x_{i}$ is mapped to a high-dimensional feature space by a nonlinear function $f(x)$, and a linear regression function $f(x)=\omega f(x)+b$ is established in the high-dimensional space, where $\omega$ and $b$ are the weight vector and the deviation, respectively. The optimization problem is expressed as follows:

$$
\left\{\begin{array}{l}
\min J(\omega, e)=\frac{1}{2} \omega^{T} \omega+\frac{1}{2} \gamma \sum_{i=1}^{n} e_{i}^{2}, \\
\text { s.t. } y_{i}=\omega^{T} \phi\left(x_{i}\right)+b+e_{i},
\end{array}\right.
$$


where $J(\omega, e)$ is the structural risk and $\gamma$ is the adjustment parameter, which is used to adjust the balance between promotion ability and empirical risk. $e_{i} \in R$ is the error variable. The Lagrange method is adopted to solve the optimization of equation (11), and the following linear equations are obtained according to the KKT optimization conditions:

$$
\left[\begin{array}{cc}
0 & E^{T} \\
E & \Omega+\gamma^{-1} I
\end{array}\right]\left[\begin{array}{l}
b \\
\alpha
\end{array}\right]=\left[\begin{array}{l}
0 \\
y
\end{array}\right]
$$

where $\quad E=[1,1, \cdots, 1]^{T}, \quad y=\left[y_{1}, y_{2}, \cdots, y_{n}\right]^{T}, \quad \Omega_{i, j}=$ $\phi\left[x_{i}\right]^{T} \phi\left[x_{j}\right]$, and $\alpha=\left[\alpha_{1}, \alpha_{2}, \cdots, \alpha_{n}\right]^{T}$ is Lagrange factor.

According to the Mercer condition, the following kernel function is defined:

$$
K\left(x_{i}, x_{j}\right)=\phi\left[x_{i}\right]^{T} \phi\left[x_{j}\right] .
$$

The regression model of LSSVM is finally derived:

$$
f(x)=\sum_{i=1}^{m} \alpha_{i} K\left(x, x_{i}\right)+b,
$$

where $m$ is the number of input samples, $x_{i}$ is the $i$-th input sample, and $x$ is a certain input variable. Selecting different kernel functions can build different LSSVM models, and the performance of the models is also different. The most widely applied radial basis kernel functions are chosen as follows in this paper:

$$
K\left(x, x_{i}\right)=\exp \left(-\frac{\left\|x-x_{i}\right\|^{2}}{2 \sigma^{2}}\right), \quad \sigma>0,
$$

where $\sigma$ is the kernel function width.

3.2. Improved Particle Swarm Optimization Algorithm (IPSO). PSO algorithm considers individuals as particles moving at a specific velocity. The velocity is dynamically adjusted according to the optimal history of itself and the entire community, and the position is updated according to the adjusted velocity until the optimal solution is found $[17,23]$. In the $m$-dimensional search space, the entire community $X$ is composed of $n$ particles, $X=\left\{x_{1}, x_{2}, \ldots, x_{n}\right\}$. The position and velocity of $i$-th are $x_{i}=\left(x_{i 1}, x_{i 2}, \ldots x_{i m}\right)^{T}$ and $v_{i}=\left(v_{i 1}, v_{i 2}, \ldots v_{i m}\right)^{T}$, respectively. The historical optimal position is $P_{i}=\left(p_{i 1}, p_{i 2}, \ldots, p_{i m}\right)^{T}$, the historical optimal position of the entire community is $P_{g}=\left(p g_{1}, p g_{2}, \ldots, p g_{m}\right)^{T}$. The particles update velocity and position according to equations (16) and (17). After multiple iterations are completed, the individual finally achieves optimization in free space:

$$
\begin{aligned}
v_{i d}(t+1)= & \omega v_{i d}(t)+c_{1} r_{1}\left[p_{i d}-x_{i d}(t)\right] \\
& +c_{2} r_{2}\left[p_{g d}-x_{g d}(t)\right], \\
x_{i d}(t+1)= & x_{i d}(t)+v_{i d}(t+1),
\end{aligned}
$$

where $\omega$ is inertia weight, $r_{1}$ and $r_{2}$ are random numbers in the range $[0,1]$, and $c_{1}$ and $c_{2}$ are learning factors.

In PSO algorithm, each particle is close to the individual and global extremum, which easily causes all particles to gather to a certain extreme point and stagnate, resulting in a decrease in convergence. Reference [17] proposed an s-dPSO optimization algorithm, which updated each dimension of the particle in each iteration, and evaluated the updated fitness. The new position of $i$-th particle is as follows:

$$
x_{i}^{\prime}(t)=x_{i 1}(t)+x_{i 2}(t) \cdots\left(x_{i d}(t)+v_{i d}(t+1)\right) \cdots v_{i m}(t+1) .
$$

After updating all dimensions of the particle, $x_{i}(t+1)=x_{i}(t)$. Since each dimension is updated based on the fitness value before the update, the new particle values are the optimal values since the iteration, and the new velocity is updated by the following equation:

$$
v_{i d}(t+1)=\omega v_{i d}(t)+c_{1} r_{1}\left(p_{g d}(t)-x_{i d}(t)\right) .
$$

The aggregation degree of the particles is described by the dimensional diversity $h(j)$ :

$$
h(j)=\sqrt{\frac{1}{n} \sum_{i=1}^{n}\left(x_{i, j}-\bar{x}_{j}\right)^{2} .}
$$

The smaller the $h(j)$, the worse the diversity and the more particles aggregate in this dimension. $h(j)$ is sorted in order, and the particles in front are mutated: redefine the value of particles in this dimension and break up the aggregation state of all particles in this dimension. The flowchart of optimizing LSSVM parameters with the IPSO algorithm is displayed in Figure 1.

The steps of optimizing LSSVM parameters with IPSO are as follows:

(1) Normalized preprocessing sample set.

(2) Set initial values of the IPSO algorithm parameters, including the inertia weight $\omega$, the number of particles in the population $N$, the learning factors $c_{1}$ and $c_{1}$, and the maximum number of iterations.

(3) Calculate the fitness value of the particles.

(4) Calculate and update the optimal fitness of individuals and the population.

(5) Optimize the velocity and position of particles according to equations (18) and (19).

(6) Determine whether the termination condition is met. If it has been met, the operation is ended and the result is output; otherwise, it returns to (3) to continue.

(7) Substitute the obtained optimal values of the penalty factor and the kernel function parameter $\sigma$ into the LSSVM regression prediction model, input training samples, and start training.

(8) Determine whether the number of iterations has been met; if it has been met, end; otherwise, return to (7).

3.3. Establishment of the IPSO- LSSVM Prediction Model. The specific process of the IPSO-LSSVM prediction model for the gas outburst is as follows: 


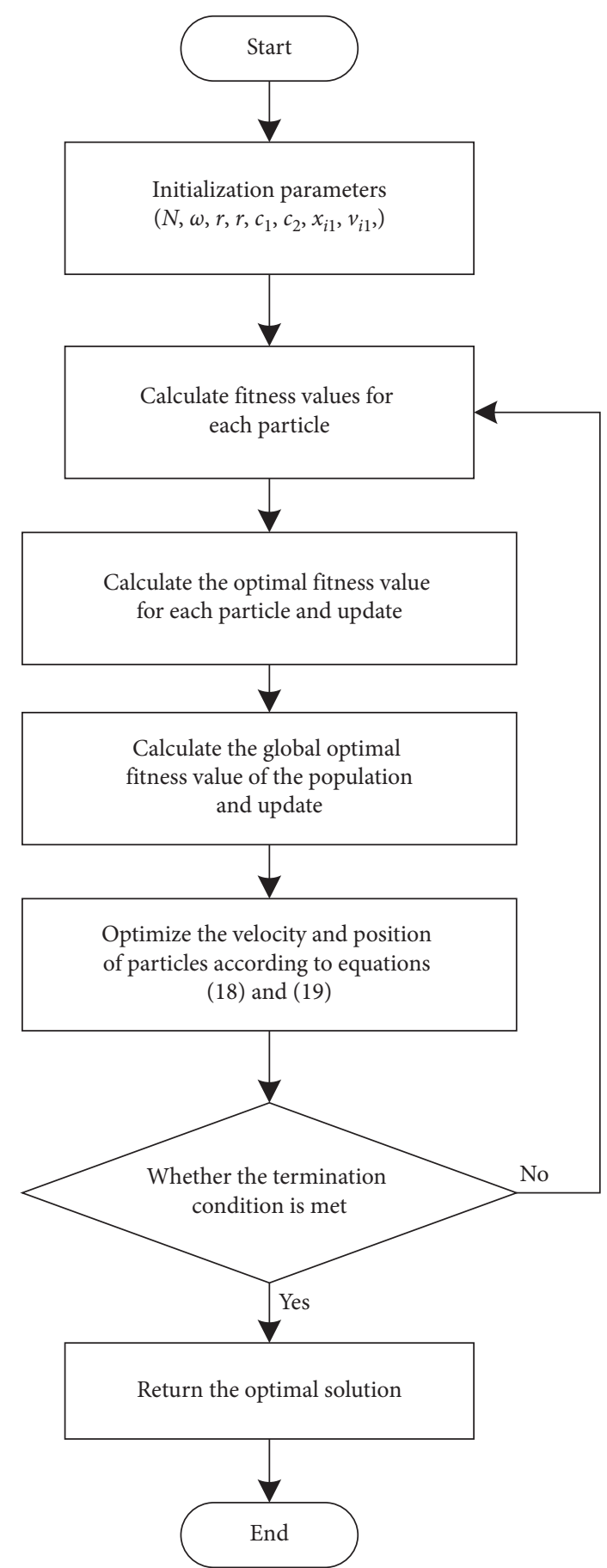

FIGURE 1: Flowchart of optimizing the LSSVM parameters with IPSO.

Step 1. Collect data for the prediction area.

Step 2. Divide the collected data into reference columns and comparative columns, analyze the data with the improved entropy weight grey correlation analysis, sort by the correlation degree, and use the prediction model to initially train and predict the factors constrained by different correlations. The dimension of the parameters is optimized according to the prediction results.
Step 3. Part of the selected parameter data is selected as the training samples and the other is test samples.

Step 4. Optimize the two parameters of LSSVM through IPSO and find the optimal combination of $c$ and $\sigma$.

Step 5. Put the optimized $c$ and $\sigma$ parameters into the LSSVM model for training.

Step 6. Substitute the test samples into the trained model and evaluate the errors and fitting degrees of the prediction results and the actual results.

The flowchart of the IPSO-LSSVM model of gas outburst prediction is displayed in Figure 2.

\section{Results and Discussion}

4.1. Extraction of Factors Influencing Gas Outburst. The mechanism of gas outburst in the working face is complicated, which is the result of a combination of factors such as geostress, coal seam gas, and physical and mechanical properties of the coal body [5]. Based on the historical data of gas outburst accidents and consulting relevant references $[2-4,24]$, this paper selects failure type of coal $\left(X_{1}\right)$, initial velocity of gas emission $\left(X_{2}, \mathrm{~mL} / \mathrm{s}\right)$, coal solidity coefficient $\left(X_{3}\right)$, gas content $\left(X_{4}, \mathrm{~m} 3 / \mathrm{t}\right)$, drill cuttings amount $\left(X_{5}, \mathrm{~kg} /\right.$ $\mathrm{m})$, gas pressure $\left(X_{6}, \mathrm{MPa}\right)$, depth of coal mining $\left(X_{7}, \mathrm{~m}\right)$, coal seam thickness $\left(X_{8}, \mathrm{~m}\right)$ as the influencing factors for the occurrence of outbursts. The amount of coal (rock) outburst is used as the basis for classification usually. According to the actual situation of the site, the outburst intensity has 4 prediction results: I (0 1t), II (1-50t), III (50 100t), and IV (100t or more). Among them, I represents no outburst, II represents small outburst, III represents medium outburst, and IV represents large-scale outburst $[25,26]$.

The data sets for prediction is from the No. 4 coal seam of Jiuli Hill mine in Jiaozuo City, China. There are 6 minable coal seams, and the coal produced is mainly lean coal and anthracite. Due to the high gas content and many structures near the working face, the coal seam has more outbursts. In the grey correlation analysis between the outburst risk and the influencing factors, the outburst risk is taken as the reference columns and the influencing factors as the comparison columns. Select the influencing factors in 60 data sets of field engineering cases in Jiuli Hill mine as sample data and establish the sample matrix, as shown in Table 1.

Based on the improved entropy weight grey correlation analysis algorithm, standardize the initial data by employing equations (2) and (3), and thus calculate the entropy weight of each influencing factor by employing equations (4) and (6). The results are shown in Table 2.

Table 2 shows that the initial velocity of gas emission has the largest weight in all the influencing factors, which also indicates that the initial velocity of gas emission $\left(X_{2}\right)$ provides the most useful information for the prediction of gas outburst.

In order to analyze and calculate the correlation between influencing factors and outburst risk in coal and gas outburst prediction, the reference columns were selected as the type of gas outburst, and the comparison columns were selected 


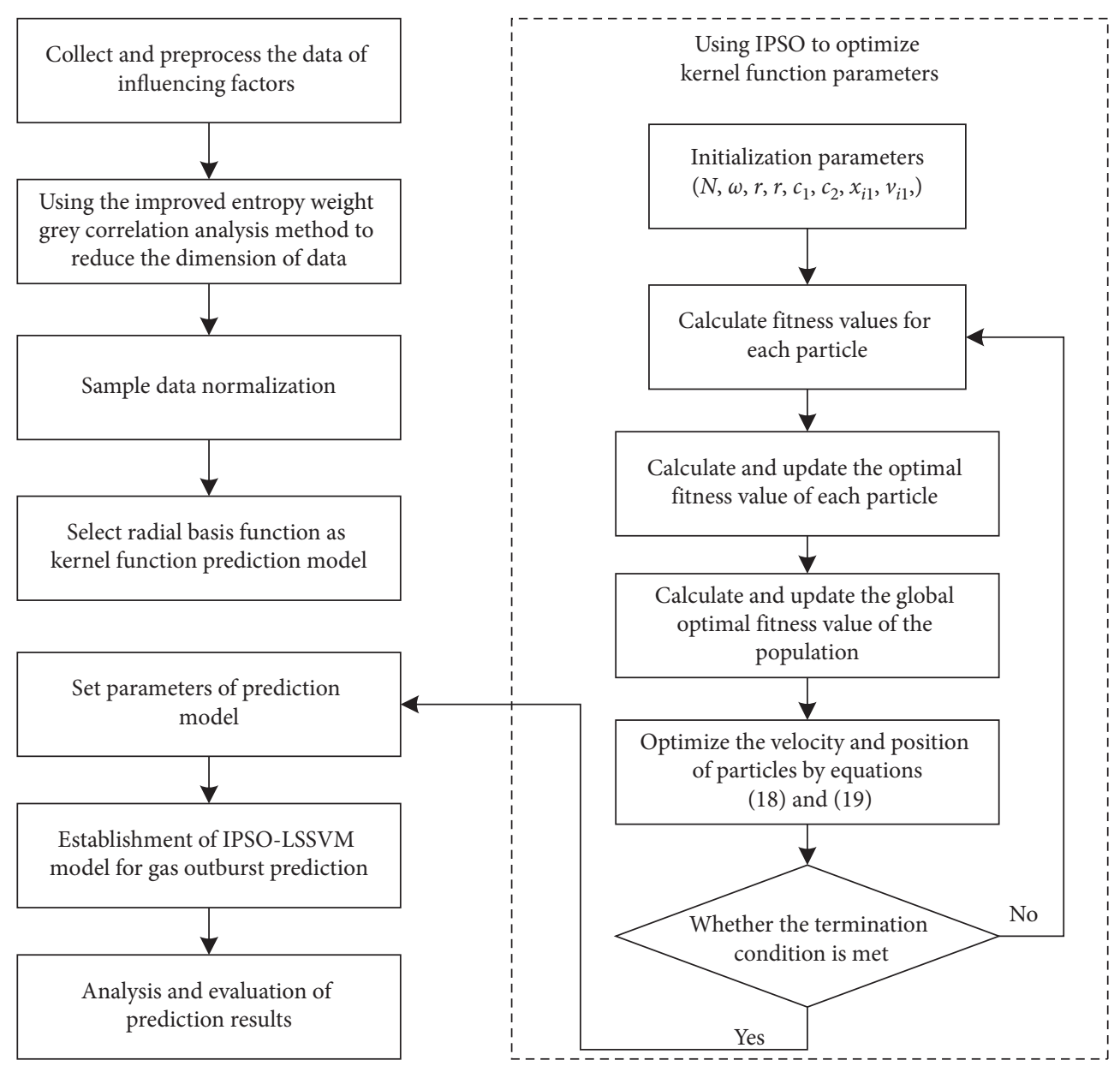

FIgURE 2: The flowchart of the IPSO-LSSVM prediction model.

Table 1: Part of the initial data on influencing factors.

\begin{tabular}{lccccccccc}
\hline \multirow{2}{*}{ Number } & & \multicolumn{3}{c}{ Comparison columns } & \multicolumn{3}{c}{ Reference columns } \\
& $X_{1}$ & $X_{2}$ & $X_{3}$ & $X_{4}$ & $X_{5}$ & $X_{6}$ & $X_{7}$ & $X_{8}$ & $X_{0}$ \\
\hline 1 & 3.00 & 16.48 & 0.36 & 11.12 & 3.00 & 0.95 & 368.00 & 2.10 & III \\
2 & 3.00 & 15.67 & 0.36 & 10.70 & 3.40 & 1.17 & 370.00 & 2.10 & III \\
3 & 3.00 & 16.21 & 0.30 & 12.90 & 3.20 & 2.76 & 370.00 & 0.00 & IV \\
4 & 3.00 & 16.55 & 0.36 & 8.75 & 3.40 & 1.12 & 368.00 & 0.00 & II \\
5 & 3.00 & 16.35 & 0.36 & 9.30 & 3.20 & 0.89 & 369.00 & 2.00 & III \\
6 & 3.00 & 17.20 & 0.36 & 8.78 & 3.40 & 2.20 & 370.00 & 2.00 & II \\
7 & 3.00 & 16.33 & 0.36 & 8.82 & 3.40 & 2.30 & 367.00 & 2.00 & III \\
8 & 3.00 & 15.32 & 0.36 & 8.35 & 3.40 & 0.76 & 365.00 & 2.60 & I \\
9 & 3.00 & 15.56 & 0.36 & 7.92 & 3.40 & 1.04 & 363.00 & 2.60 & I \\
10 & 2.00 & 16.23 & 0.28 & 10.15 & 3.60 & 1.68 & 363.00 & 2.60 & III \\
11 & 2.00 & 16.55 & 0.28 & 11.23 & 3.80 & 1.53 & 363.00 & 2.60 & III \\
12 & 2.00 & 17.52 & 0.28 & 13.45 & 3.20 & 2.89 & 360.00 & 2.30 & IV \\
13 & 3.00 & 15.85 & 0.36 & 9.80 & 3.40 & 2.72 & 360.00 & 2.30 & II \\
14 & 3.00 & 16.32 & 0.36 & 10.12 & 3.40 & 2.38 & 360.00 & 0.00 & II \\
15 & 3.00 & 15.56 & 0.36 & 7.86 & 3.60 & 1.34 & 365.00 & 2.50 & I \\
\hline
\end{tabular}

TABLE 2: Entropy weight of each influencing factor.

\begin{tabular}{lcccccccc}
\hline Influencing factor & $X_{1}$ & $X_{2}$ & $X_{3}$ & $X_{4}$ & $X_{5}$ & $X_{6}$ & $X_{7}$ & $X_{8}$ \\
\hline Entropy weight & 0.197 & 0.223 & 0.212 & 0.268 & 0.165 & 0.188 & 0.176 & 1.172 \\
\hline
\end{tabular}


TABLE 3: The grey correlation coefficient.

\begin{tabular}{lcccccccc}
\hline Number & $X_{1}$ & $X_{2}$ & $X_{3}$ & $X_{4}$ & $X_{5}$ & $X_{6}$ & $X_{7}$ \\
\hline 1 & 0.53 & 0.71 & 0.53 & 0.93 & 0.47 & 0.85 & 0.60 \\
2 & 0.53 & 0.53 & 0.53 & 0.96 & 0.96 & 0.60 & 0.53 \\
3 & 1.00 & 0.41 & 0.40 & 0.78 & 0.45 & 0.47 & 0.66 \\
4 & 0.53 & 0.73 & 0.53 & 0.62 & 0.90 & 0.60 & 0.60 \\
5 & 0.53 & 0.67 & 0.53 & 0.69 & 0.76 & 0.71 & 0.56 \\
6 & 0.53 & 0.97 & 0.53 & 0.62 & 0.58 & 0.92 & 0.53 \\
7 & 0.53 & 0.67 & 0.53 & 0.63 & 0.76 & 0.65 & 0.64 \\
8 & 0.33 & 1.00 & 0.33 & 0.72 & 0.50 & 0.74 & 0.70 \\
9 & 0.33 & 0.87 & 0.33 & 0.80 & 0.56 & 0.58 & 0.70 \\
10 & 0.47 & 0.64 & 0.47 & 0.83 & 0.90 & 0.84 & 0.44 \\
11 & 0.47 & 0.73 & 0.47 & 0.90 & 0.79 & 0.78 & 0.87 \\
12 & 0.33 & 0.60 & 0.33 & 0.89 & 1.00 & 0.90 & 0.87 \\
13 & 0.53 & 0.56 & 0.53 & 0.77 & 0.72 & 0.55 & 0.49 \\
14 & 0.53 & 0.66 & 0.53 & 0.82 & 0.90 & 0.92 & 0.53 \\
15 & 0.33 & 0.87 & 0.33 & 0.81 & 0.56 & 0.43 & 0.83 \\
\hline
\end{tabular}

TABLE 4: Results of the improved entropy weight grey correlation analysis.

\begin{tabular}{lccccccc}
\hline Influencing factor & $X_{1}$ & $X_{2}$ & $X_{3}$ & $X_{4}$ & $X_{5}$ & $X_{6}$ & $X_{7}$ \\
\hline Average grey correlation degree & 0.6971 & 0.7941 & 0.7256 & 0.8881 & 0.432 & 0.6516 & 0.5249 \\
Weighted grey correlation degree & 0.1466 & 0.1593 & 0.1524 & 0.1685 & 0.1026 & 0.1482 & 0.1223 \\
Order of correlation degree & 4 & 2 & 3 & 1 & 8 & 5 & 6.1029 \\
\hline
\end{tabular}

as the influencing factor of gas outburst. After preprocessing the data in Table 1, the grey correlation coefficient is calculated by employing equation (8), and then the average grey correlation and embedded grey entropy weight are obtained according to the operations of equations (9) and (10). The results are shown in Tables 3 and 4 .

As can be seen from Table 4, the order of the correlation degree of each influencing factor is $X_{4}>X_{2}>X_{3}>X_{1}>$ $X_{6}>X_{7}>X_{8}>X_{5}$. The weighted grey correlations of gas content and coal solidity coefficient is 0.1685 and 0.1593 , respectively, indicating that the two have the greatest impact on the danger of gas outburst. Therefore, in the practice of controlling the abovementioned factors for gas outburst prediction, the gas content and initial velocity of gas emission can be emphasized, which can produce more ideal results.

4.2. Prediction Experiment of the IPSO-LSSVM Model. This experiment was performed in MATLAB R2016b and involved 60 data sets, of which 48 were used for training and 12 for prediction. According to the results of the improved entropy weight grey correlation analysis, gas content $\left(X_{4}\right)$, initial velocity of gas emission $\left(X_{2}\right)$, coal solidity coefficient $\left(X_{3}\right)$, and failure type of coal $\left(X_{1}\right)$ were selected as the main controlling factors influencing coal and gas outburst. In order to verify the effectiveness of the prediction model in this paper, the IPSO-LSSVM model is compared with the GA-LSSVM [13] and SVM model [15]. The population optimal fitness and average fitness curves of the IPSO algorithm and GA algorithm are shown in Figure 3, respectively. The prediction results are shown in Figure 4.
As can be observed from the population fitness curves, for the IPSO algorithm, the optimal fitness value of the population began to stabilize after the $4^{\text {th }}$ generation. Although there were slight fluctuations in the subsequent evolution process, the fluctuation range was small, and it was basically stable near 0.027 . For the GA algorithm, the optimal fitness value converged to a small extent after the population evolved to the $7^{\text {th }}$ generation. There was a large convergence to the $22^{\text {nd }}$ to $23^{\text {rd }}$ generation, and the optimal fitness basically stabilized at the $27^{\text {th }}$ generation. Therefore, the convergence speed of the IPSO is significantly better than that of the GA. In addition, considering the calculation speed and simplicity of the model, the GA has disadvantages of complex structure, many parameters, slow calculation speed, and so on.

The kernel function of the LSSVM model is selected by comparing the three kernel functions and the other parameters are set at the default value of the system under the same condition. The relative error and standard deviation of the gas outburst prediction model established by the three kernel functions in the training process were compared, as shown in Table 5.

Table 5 shows that the relative error and standard deviation of the prediction model established by RBF_kernel function are less than the model established by LIN_kernel function and Poly_kernel. Therefore, RBF_kernel function is used in this study.

Figure 4 shows that the prediction results of the IPSOLSSVM model agree with the actual intensity well. The accuracy of the SVM prediction model is 67\%, the GALSSVM prediction model is $83 \%$ and that of the proposed model is $92 \%$, indicating that the IPSO-LSSVM model has 


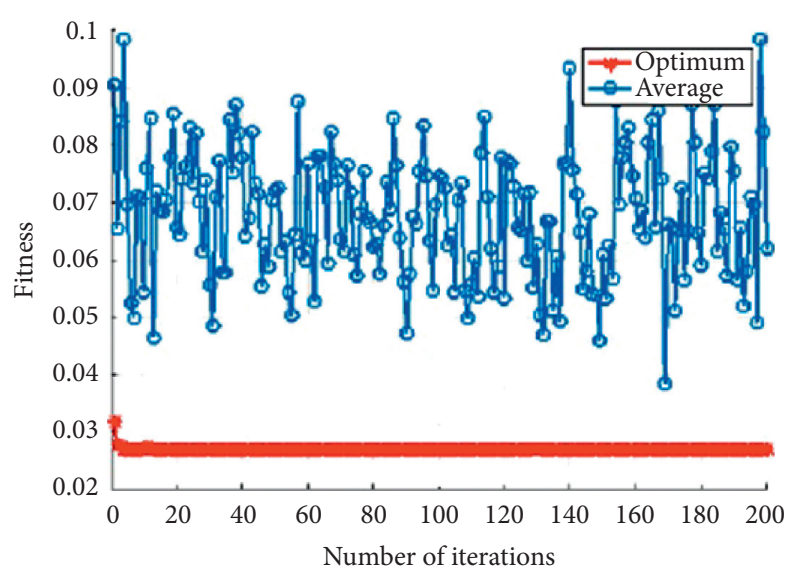

(a)

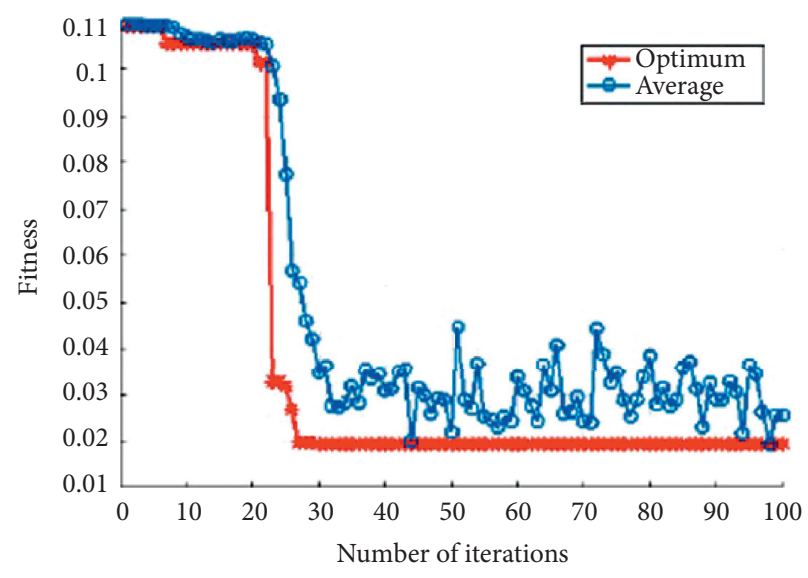

(b)

FIgURE 3: Comparison of optimum fitness and average fitness of the population of the IPSO and GA algorithm. (a) The IPSO algorithm. (b) The GA algorithm.

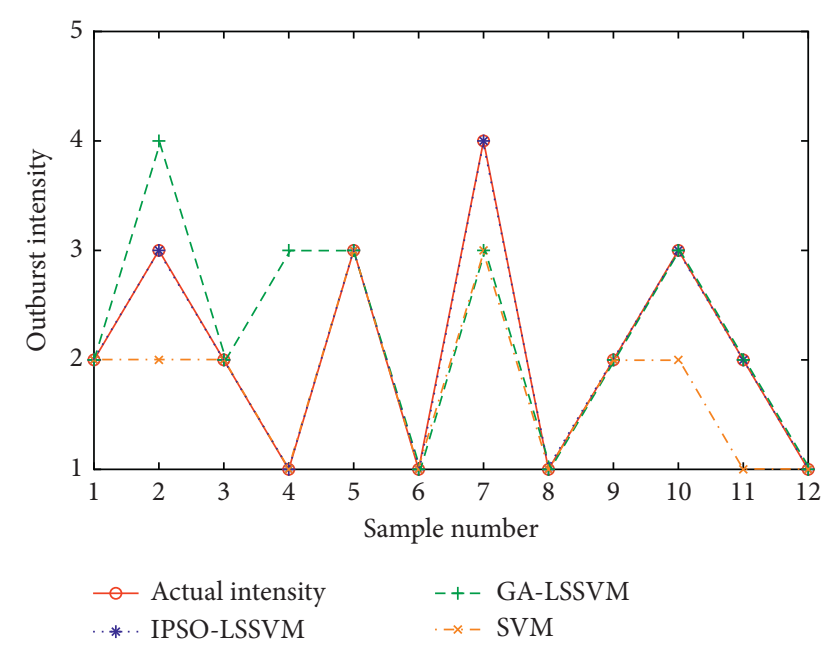

Figure 4: Prediction results of the three models.

TABLE 5: Relative error and standard deviation of three kernel functions.

\begin{tabular}{lcc}
\hline Kernel functions & Relative error \% & Standard deviation \% \\
\hline POLY_kernel & 0.158 & 0.149 \\
LIN_kernel & 0.093 & 0.076 \\
RBF_kernel & 0.072 & 0.059 \\
\hline
\end{tabular}

higher accuracy. Figure 5 shows that the average relative errors of the three prediction models are $2.0 \%, 5.8 \%$, and $9.0 \%$, respectively. The IPSO-LSSVM model is the smallest, followed by the GA-LSSVM model. Both the IPSO-LSSVM model and the GA-LSSVM model have certain optimization effects, but the IPSO-LSSVM model shows higher adaptability. For the SVM model, the combination of its two parameters is liable to fall into the dilemma of the local optimal solution, which affects the prediction accuracy.

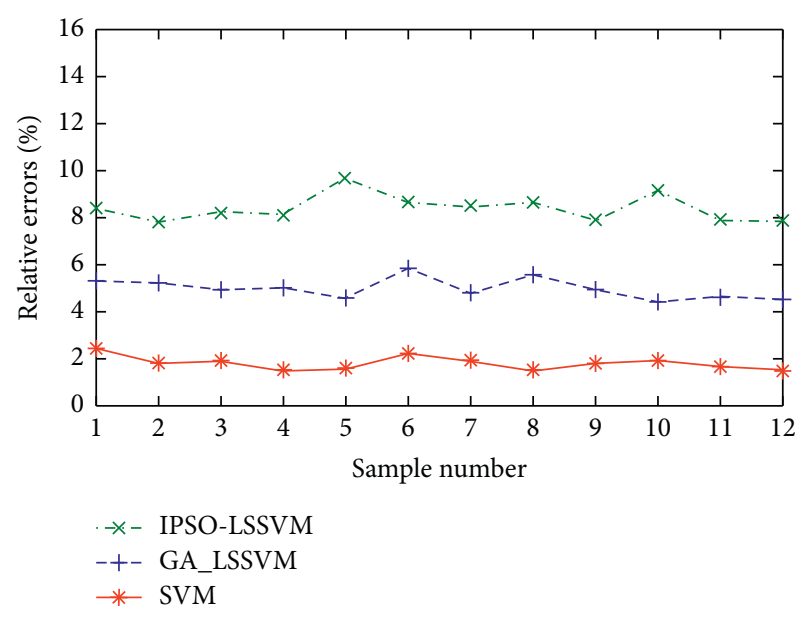

FIGURE 5: Relative errors of three models.

\section{Conclusions}

In this paper, a new prediction model of gas outburst based on the improved entropy weight grey correlation analysis and IPSO-LSSVM is established. The main conclusions are as follows:

(1) The improved entropy weight algorithm is introduced into a grey correlation analysis algorithm to identify the main influencing factors. The reasonable weights and correlation order of influencing factors are obtained to avoid the subjectivity of weight determination and reduce the fluctuation of correlation coefficients.

(2) By using IPSO to optimize the punishment factor and nuclear radial range of LSSVM, the optimization enhances the global search ability and convergence speed of the entire system. The gas outburst prediction model based on IPSO-LSSVM is established, which can improve the accuracy of gas outburst prediction. 
(3) The IPSO-LSSVM model proposed in this paper is applied in the tunneling heading face 14141 of Jiuli Hill mine in Jiaozuo City, China. The case study demonstrates that the IPSO-LSSVM model has higher accuracy than the SVM model and the GALSSVM model. It should be mentioned that although the proposed prediction model is proved to be more accurate, gas outburst is a complex nonlinear dynamic process affected by many factors. There are still some interesting issues that need to be studied, such as gas outburst mechanism and application of some newly developed methods.

\section{Data Availability}

The data used to support the findings of this study are available from the corresponding author upon request.

\section{Conflicts of Interest}

The authors declare that there are no conflicts of interest regarding the publication of this paper.

\section{Acknowledgments}

This research was supported by the National Science Foundation of China (51604096), Key Project of Science and Technology of Education Department of Henan Province (19B120002), and Key Laboratory of Control Engineering of Henan Province (KG2016-17).

\section{References}

[1] P. Guan, H. Wang, and Y. Zhang, "Mechanism of instantaneous coal outbursts," Geology, vol. 37, no. 10, pp. 915-918, 2009.

[2] H. Rong, H. W. Zhang, and B. Liang, "Instability mechanism of dynamic system of coal and rock," Journal of China Coal Society, vol. 42, no. 7, pp. 1663-1671, 2017.

[3] D. Li, S. P. Peng, and W. F. Du, "Comprehensive prediction method of coal seam gas outburst danger zone," Journal of China Coal Society, vol. 43, no. 2, pp. 466-472, 2018.

[4] B. S. Nie, "Present situation and progress trend of prediction technology of coal and gas outburst," China Safety Science Journal, vol. 13, no. 6, pp. 40-43, 2003.

[5] L. S. Shao and G. X. Fu, "Identification and decision of coal mine gas information based on data mining," Journal of Liaoning Technical University, vol. 27, no. 2, pp. 288-291, 2008.

[6] M. He, "A gas outburst prediction model based on data mining and information fusion," Revue d'Intelligence Artificielle, vol. 33, no. 5, pp. 379-386, 2019.

[7] H. W. Zhang, J. Han, and W. H. Song, "Prediction of coal and gas outburst based on PCA-BP neural network," China Safety Science Journal, vol. 23, no. 4, pp. 46-51, 2013.

[8] H. B. Liu, Y. B. Li, and F. Z. Wang, "Evaluation strategy of gas outburst based on fuzzy neural network and evidence theory," Journal of University of Shanghai for Science and Technology, vol. 38, no. 2, pp. 168-171, 2016.

[9] N. Li, L. G. Wang, and M. T. Jia, "Rock burst prediction based on rough set theory and support vector machine," Journal of
Central South University (Science and Technology), vol. 48, no. 5, pp. 1268-1275, 2017.

[10] R. L. Zhang and I. S. Lowndes, "The application of a coupled artificial neural network and fault tree analysis model to predict coal and gas outbursts," International Journal of Coal Geology, vol. 84, no. 2, pp. 141-152, 2010.

[11] D. Rudakov and V. Sobolev, "A mathematical model of gas flow during coal outburst initiation," International Journal of Mining Science and Technology, vol. 29, no. 5, pp. 791-796, 2019.

[12] M. Yang, J. W. Yun, and P. C. Yuan, “An incorporate genetic algorithm based back propagation neural network model for coal and gas outburst intensity prediction," Procedia Earth «Planetary Science, vol. 1, no. 1, pp. 1285-1292, 2009.

[13] Z. H. Wang and N. Qiao, "Prediction model of coal and gas outburst intensity based on IGA-LSSVM," Journal of Liaoning Technical University: Natural Science, vol. 34, no. 7, pp. 791796, 2015.

[14] H. B. Liu, W. Qian, and F. Z. Wang, "Gas outburst prediction based on rough set and particle swarm optimization support vector machine," Journal of University of Science and Technology of China, vol. 49, no. 2, pp. 87-92, 2019.

[15] M. Qian, X. P. Ma, and Y. Zhou, "Forecasting of coal seam gas content by using support vector regression based on particle swarm optimization," Journal of Natural Gas Science and Engineering, vol. 21, pp. 71-78, 2014.

[16] W. B. Qiao, Z. Yang, and Z. Y. Kang, "Short-term natural gas consumption prediction based on Volterra adaptive filter and improved whale optimization algorithm," Engineering Applications of Artificial Intelligence, vol. 87, pp. 1-19, 2020.

[17] W. B. Qiao and Z. Yang, "Forecast the electricity price of US using a wavelet transform-based hybrid model," Energy, vol. 193, pp. 1-20, 2020.

[18] T. X. Wen, F. E. Yu, and L. S. Shao, "Probabilistic neural network prediction model of coal and gas outburst based on grey relational entropy," Application Research of Computers, vol. 35, no. 11, pp. 3326-3329, 2018.

[19] L. Huang, W. W. Du, and L. X. Ding, "Particle swarm optimization algorithm based on adaptive sigmoid inertia weight," Application Research of Computers, vol. 29, no. 1, pp. 32-34, 2012.

[20] Y. Wu, R. Gao, and J. Yang, "Prediction of coal and gas outburst: a method based on the BP neural network optimized by GASA," Process Safety and Environmental Protection, vol. 133, pp. 64-72, 2020.

[21] Y. Chen, C. Gu, C. Shao et al., "An approach using adaptive weighted least squares support vector machines coupled with modified ant lion optimizer for dam deformation prediction," Mathematical Problems in Engineering, vol. 2020, pp. 1-23, 2020.

[22] H. R. Zhang, Y. Yang, and Y. Zhang, "A combined model based on SSA, neural networks, and LSSVM for short-term electric load and price fore casting," Neural Computing \& Applications, 2020.

[23] Z. X. Chen and D. Wang, "A prediction model of forest preliminary precision fertilization based on improved GRAPSO-BP neural network," Mathematical Problems in Engineering, vol. 2020, pp. 1-17, 2020.

[24] Y. Liang, D. Guo, Z. Huang, and X. Jiang, "Prediction model for coal-gas outburst using the genetic projection pursuit method," International Journal of Oil, Gas and Coal Technology, vol. 16, no. 3, pp. 271-281, 2017.

[25] G.-Y. Wei, F.-C. Kang, B.-B. Qin, T.-R. Jia, J.-W. Yan, and Z.-D. Feng, "A novel method for evaluating proneness of gas 
outburst based on gas-geological complexity," Natural Hazards, vol. 104, no. 2, p. 1841, 2020.

[26] C. He, X. Liu, and J. Liu, "Risk analysis of gas outburst tunnel construction based on the fuzzy comprehensive evaluation method," Electronic Journal of Geotechnical Engineering, vol. 19, pp. 8643-8654, 2014. 